\title{
The Basic Query Machine of the KIWIS system $\ddagger$
}

\author{
N. Leone, A. Mecchia, G. Rossi, P. Rullo \\ CRAI - Loc. S. Stefano, 87036 Rende (Italy)
}

\begin{abstract}
KIWIS is an advanced environment for large database systems which supports knowledge-based applications and provides a seamless integration of information coming from different external sources. The system relies on a new knowledge representation and manipulation language, called LOCO, that is based on a tight integration between the object-oriented and the logic programming paradigm. The implementation of LOCO is supported both by a top-down and a bottom-up executor, allowing great flexibility and suitability for large knowledge based applications. This paper describes the basic implementation principles as well as the integrated architecture of the Basic Query Machine (BQM) - the module of KIWIS in charge of implementing the bottom-up model of execution of a class of LOCO programs. The structure of such programs (called stratified), amenable of an efficient bottom-up evalutation even in case of data intensive applications, is discussed in details. The mechanism for a strict cooperation with the top-down evaluator, is also presented.
\end{abstract}

\section{Introduction}

KIWIS is an advanced environment for large database systems which supports knowledge-based applications and provides a seamless integration of information coming from different external sources ([KIWIS89,VL90,Ah191]).

The system relies on a new knowledge representation and manipulation language, called LOCO [LVVS90], that is based on a tight integration between the objectoriented and the logic programming paradigm. In addition, the language supports features such as defeasible and default reasoning, making it suitable also for AIflavored applications (e.g. expert systems).

The overall architecture of the system is shown in Figure 1.1.

The Abstraction Layer (AL) and the Basic Query Machine (BQM) [LMRR91] provides the necessary support for the native KIWIS language, LOCO, by implementing respectively backward (top-down) and forward (bottom-up) chaining. The rationale of having two different execution strategies is twofold: the bottom-up engine ensures the proper termination of fully declarative LOCO programs, granting an efficient execution also with large amount of data; on the other hand, the top-down evaluator supports the execution of general LOCO programs (even if termination is not granted), including updates, metaprogramming and extra-logical predicates. Thus, the coexistence of top-down and bottom-up engines becomes one of the relevant features of the KIWIS system, allowing great flexibility, suitability for large knowledge based

\# Research partially supported by EEC in the framework of ESPRIT II project EP2424 "KIWIS". 


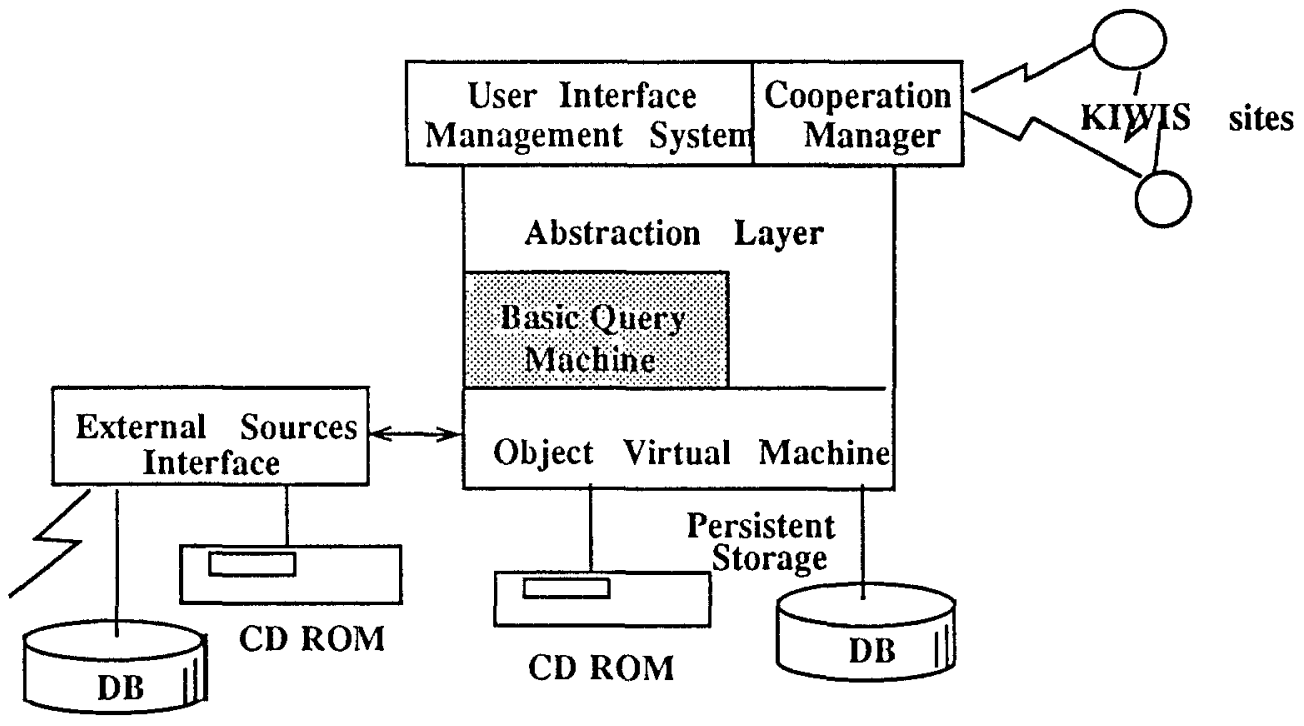

Figure 1.1: Overall KIWIS Architecture

applications.

Both $\mathrm{AL}$ and $\mathrm{BQM}$ can rely on the Object Virtual Machine (OVM) for the efficient management of complex objects both in main and secondary memory. On the other hand, based on the AL and BQM services, the User Interface Development System (UIDS) supports customizable views on the data together with several interaction paradigms of increasing complexity [SLT90,STVDT91].

Finally, two kinds of "hooks" to external information sources represent a valuable channel for the import, export, and (re-)usage of existing data.

In one case, the External System Interface (ESI) provides an extendible, readonly interface to a variety of external information sources (e.g. traditional, text or picture databases and applications, possibly residing on remote systems). Alternatively, the Cooperation Manager (CM) allows to define KIWIS as a federation [AJ90] of loosely coupled (KIWIS) knowledge bases, to form a network for information sharing and cooperation without a commitment to a centrally maintained global schema.

The aim of this paper is to describe the design of the BQM (as bottom-up machine) and its interaction with the AL (as top-down machine). We start, in the next Section, with an informal description of the query capabilities supported by LOCO. Then, Section 3 presents the BQM language, i.e. a class of LOCO programs (indicated as stratified) that are amenable of an efficient boltom-up evaluation. Finally, Section 4 illustrates the relevant aspects of the actual $\mathrm{BQM}$ architecture. For details on some basic implementation principles, the reader is referred to the Appendices.

\section{Language Overview}

This section gives an informal presentation of the main features of a (fully declarative) subset of the LOCO language. 
As already pointed out, LOCO is a fresh attempt of enhancing the logic programming paradigm with some of the most relevant Object Oriented (OO) features [LVVS90]. From the deductive approach, LOCO maintains a clearly defined semantics along with declarative query capabilities. On the other hand, from the $O O$ field, the notions of object identity, structured object, and (multiple) inheritance are formally accomodated into the language as first class features. On top of this, non-monotonic and default reasoning are introduced in the language following some recent proposals [GL90,GS90,KS90,LSV90] on negative logic programs (i.e. allowing negation in the head of rules).

The language presented here, that we call Extended Ordered Logic (EOL), fully captures the query capabilities of the LOCO language and is a direct evolution of the Ordered Logic (OL) programming. The main extention of EOL with respect to OL boils down to a sort of message passing mechanism. More formal and detailed discussions about OL can be found in [LSV90,LV90,Lae90,LR91].

As a very basic starting point, an EOL program consists of a number of objects, each one identified by a (unique) name and composed of a set of rules possibly with negative head literals. We briefly recall that a rule is a statement of the form $L_{0} \leftarrow L_{1}, \ldots, L_{n}$, where $L_{0}, L_{1}, \ldots, L_{n}$ are literals. $L_{0}$ is the head literal while the set of literals $L_{1}, \ldots, L_{n}$ constitutes the body of the rule. A rule with an empty body is also called $f a c t$. A positive (negative) literal has the format $p(X)(\neg p(X))$ where $X$ is a list of arguments and $p(\neg p)$ is a literal symbol. We will often denote simply by $p$ a generic literal symbol, either positive or negative.

Example 2.1. (objects)

$$
\begin{aligned}
\text { fred }=\left(\begin{array}{l}
\text { name }(" F r e d ") . \\
\text { married :- wife }(X) . \\
\text { wife }(\text { sally }) .
\end{array}\right\}
\end{aligned}
$$

fred, is the object identifier; the literal symbols name, married, and wife are used to define the properties of fred. In particular, the facts name ("Fred"), and wife (sally) hold along with the property married which is inferred by the rule married :- wife $(X)$. No negative literals appear in fred.

In short, an object is a logical theory or, mainly according to personal taste and background, one might imagine facts and rules instead of, e.g., instance variables and methods, respectively. In the above example, name could be seen as an instance variable whose value is Fred, and married as a boolean instance variable. Further, the rule married :- wife $(X)$ might represent a method for 'computing' the property married.

Inheritance is a central characteristic of the EOL language. The knowledge of an object is not confined exclusively to its own rules: a simple and general specificity relation (sub/super-object) is used to model how rules filter down from one object to another.

Example 2.2. (inheritance) Consider the following program, where fred is now a sub-object of person. (Note: each object is preceeded by the list of its immediate super-objects). 


$$
\begin{aligned}
\text { person }=\left\{\begin{array}{l}
\text { married :- wife }(Y) . \\
\text { fred }=\{\text { wife }(\text { sally }) .
\end{array}\right. \\
\text { name }(" F r e d ") .\}
\end{aligned}
$$

It should be intuitively clear that the properties of fred have not changed from the previous example. The rule for married is inherited from person so that married can be inferred for fred. On the other hand, no property holds for person as it misses the necessary facts for any valid inference.

Concluding, each object has its own perception of the knowledge base, given by its local rules plus some global rules, that is, those of the objects that are connected to it via the sub-object links. However, it is possible for an object, to refer explicitely to the properties of another object. This is achieved by allowing, within rules' bodies, extended literals of the form $X . p$, where $X$ is either an object identifier or a variable and $p$ is a literal. Intuitively, an extended literal $X . p$ should be read as " $p$ is true at object $X$ " or "the property $p$ of the object $X$ ".

Example 2.3. (extended literals)

$$
\begin{aligned}
& \text { fred }=\{\text { wife (sally). } \\
& \text { child (john). I } \\
& \text { sally }=\{\text { husband (fred). } \\
& \text { john }=\{\} \\
& \text { child }(X) \text { :- husband }(Y), Y \text {.child }(X) \text {. I }
\end{aligned}
$$

The program describes a married couple, fred and sally, with only one child, john. Instead of declaring explicitely john as sally's child, we use a rule with an extended literal. The rule child $(X)$ :- husband $(Y), Y$.child $(X)$. says that for each husband $Y$ of sally, the child $X$ of $Y$ is also a child of sally. Thus, since husband (fred) holds in sally and child (john) is true at object fred, it is possible to infer child (john) for sally.

Notice how the extended literals mimic the message passing mechanism of object-oriented languages; indeed, $Y$.child $(X)$ can be seen as a message to all $Y$ objects, asking for the value of the child property. Consistently, a query to an EOL program is an extended literal, e.g. sally.child $(X)$ asks for all $X$ values of the child property for sally.

Let us now turn our attention to the treatement of contradictions. As we shall see, contradictions are mastered by two simple rules, namely overruling and defeating, driven by the relationships between the involved objects.

Example 2.4. (overruling)

$$
\begin{array}{lll}
\text { person } & =\{\text { taxPayer. }\} \\
\text { (person) } & \text { unemployed } & =\{- \text { taxPayer. }\} \\
(\text { unemployed }) & \text { tom } & =\{1 \\
\text { (person }) & \text { john } & =\{1
\end{array}
$$

The property taxPayer acts as a "default": it is visible from all person's sub-objects and it holds unless explicitely contradicted. In fact, taxPayer is true for person and for john. On the contrary, only the property $\neg$ taxPayer holds for tom and unemployed, and the property taxpayer is rejected or, as we say, overruled. The rationale 
of such a preference rule is that more specific information is certainly more reliable than inherited, more general properties.

Unlikely the situation above, where the ordering between the involved objects suggests an intuitive solution to contradiction handling, there are many real-world situations that are intrinsically ambiguous. The well known Nixon Diamond example [Tou86], shown next, illustrates a case of ambiguity coming from the multiple inheritance.

Example 2.5. (defeating)

$$
\begin{aligned}
& \text { quaker }=\{\text { pacifist. }\} \\
& \text { republican }=\{\rightarrow \text { pacifist. }\} \\
& \text { (quaker republican) nixon }=\{\}
\end{aligned}
$$

The program states: quakers are pacifist, republicans are not pacifist, nixon is both a quaker and a republican. Now, consider the question: is nixon a pacifist ? There is no correlation between republican and quaker and, actually, no indication of which point of view should be thrusted: the network is perfectly ambiguous and both conclusions are rejected or, as we say, defeated.

The above examples can be exploited for a further comment on the sub/superobject relation. As a matter of fact, it turns out that such a relation is able to capture both classification and generalization hierarchies. For instance, in the Example 2.4 the objects person and unemployed could be viewed as a sort of class objects, whereas john and tom constitute the class population of resp. person and unemployed; hence, tom could be considered an instance-of unemployed, while unemployed IS-A person.

It is worth noting that the language actually supports true negation instead of negation by failure, i.e., a negative fact can be inferred only if explicitely derived by a rule of the program, so that any negative information is as much valuable as a positive one.

Example 2.4. (true negation)

$$
\begin{aligned}
& \text { person }=\{\text { taxPayer. }\} \\
& \text { (person) tom }=1 \neg \text { taxPayer :- - } \text { rich. } 1
\end{aligned}
$$

The meaning of this program is that tom is a taxpayer. The reason is that the failure to prove rich is not sufficient to infer $\neg$ rich and, consequently, $\neg$ taxPayer.

\section{Stratified programs}

For clarity's sake, in this section we restrict ourselves to EOL programs where no extended literal appears. Such programs are known in literature as Ordered Logic programs [LSV90,LV90,Lae90,LR91] (OL programs, for short). Then, we single out a meaninful class of OL programs, that we call stratified programs. The extension of the notion of stratified program to cope with the presence of extended literals is rather immediate and it is discussed in Appendix 1.

A formal semantics for OL, based on an extension of the well-founded semantics of traditional logic programming [VGRS88], has been defined in [LMRR91, LR91]. In 
particular, a bottom-up model of computation of such a semantics is given as a suitable adaptation of the algorithm presented in [Van89] for classical logic programs. Unfortunately, as in that case, it turns out that the computation of the well-founded model is quite demanding.

It is possible, however, to specialize the general method and make it very efficient for a meaningful class of programs, called stratified OL programs ([LMRR91,LR91]).

Typically, a stratified program shows a two-level structure: the definition of a property belongs to a certain object, while the exceptions (i.e. conflicting rules) belong only to its subobjects. In a sense, the notion of stratification can be intended as a way to detect when an OL program has a simple structure w.r.t. the treatment of contradiction so that its well-founded model can be computed in a monotonic fashion.

We illustrate the basic intuition by an example.

Example 3.1.

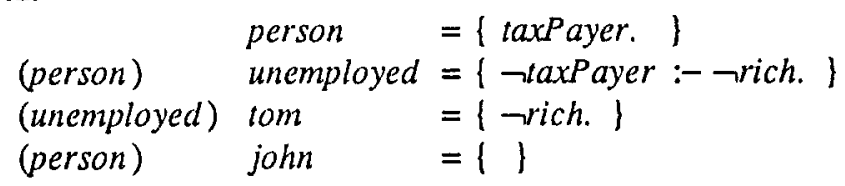

The evaluation of tom's properties could proceed as follows: first, we derive the fact $\neg$ rich; then we infer $\neg$ taxPayer from the rule $\neg$ taxPayer :- $\neg$ rich. defined in unemployed. Thereafter, taxPayer is derived through the rule in person, but, since $\neg$ taxPayer has already been inferred, taxPayer is discarded. Actually, $\neg$ taxPayer, and $\neg$ rich. are the only facts holding for tom (they are the well-founded model for tom). The reason why this approach works correctly in this case is that the above OL program has a syntactic structure ensuring the existence of a bottom-up rule evaluation ordering that preserves the "precedence" of more specific over more general rules (e.g. the precedence of the rule $\neg$ taxPayer :- $\neg$ rich. w.r.t. taxPayer.). Thus, we say that the program for tom is stratified.

Now, let $P_{o}$ denote the set of rules defined at $o$ and all its superobjects: this corresponds to the program visible by the object $o$. Given two literal symbols $q_{1}$, and $q_{2}$ appearing in $P_{o}$, we say that $q_{1}$ depends $-o n q_{2}$, denoted by $(q 1, q 2)$, if either:

1) $q_{2}$ appears in the body of a rule for $q_{1}$, or

2) $q_{1}$ and $q_{2}$ are complementary literal symbols ${ }^{1}$, defined by rules $r_{1}$ and $r_{2}$ (resp.), and $o_{d e f}\left(r_{1}\right)$ is not a sub-object of $o_{d e f}\left(r_{2}\right)$.

where $o_{d e f}(r)$ denotes the object where $r$ appears.

Example 3.2. The program $P_{\text {iom }}$ of example 3.1 is composed by the following rules: taxPayer. $\neg$ taxPayer :- $\neg$ rich. $\neg$ rich.

The depends-on relation for such a program gives ( $\neg$ taxPayer, $\neg$ rich) by condition 1 ), and (taxPayer, $\neg$ taxPayer $)$ by condition 2$)$, as $o_{d e f}($ taxPayer. $)=$ person is not a subobject of $o_{\text {def }}(\neg$ taxPayer $: \neg$ rich $)=$ unemployed .

\footnotetext{
1 i.e., either $q_{1}=\neg q_{2}$ or $q_{2}=\neg q_{1}$.
} 
Let us now consider the program $P_{\text {nixon }}$ of example 2.5. The depends-on relation gives: ( $\neg$ pacifist,pacifist) and (pacifist, $\neg$ pacifist) as quaker is not a sub-object of republican and viceversa.

At this point we can give the definition of dependency graph for an object $o$. Given the program $P_{o}$ for $o$, the associated dependency graph $D G_{o}$ is a directed graph such that: i) the nodes are the literal symbols appearing in $P_{o}$, and ii) there is an arc from node $q_{1}$ to $q_{2}$ iff $q_{1}$ depends-on $q_{2}$.

Figure 3.1 shows the dependency graphs for the objects tom of Example 3.1, and nixon of Example 2.5.
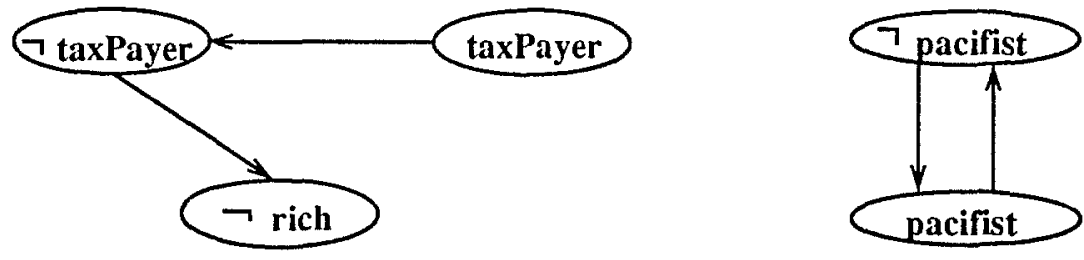

Figure 3.1: $D G_{\text {tom }}$ and $D G_{\text {nixon }}$

Hence, the dependency graph associated to an object $o$ is a compact representation of the $\mathrm{KB}$, as it is perceived by $o$. Now, let us denote by $D G_{o . p}$ the subgraph of $D G_{o}$ that consists of the node $p$ and all nodes that are reachable from $p$ (in $D G_{o}$ ). We denote by $P_{o . p}$ the program consisting of all rules (of $P_{o}$ ) whose head literals label the nodes of $D G_{o . p}$. Thus, a program $P_{o . p}$ is stratified if no cycle of $D G_{o . p}$ contains two complementary literal symbols.

Example 3.4 Consider the following program:

$$
\begin{aligned}
& \text { person }=\{\text { taxPayer } \text {. } \\
& \text { citizen :- taxPayer. } \\
& \text {-citizen :- alien. } \mid \\
& \text { (person) unemployed }=\{\neg \text { taxpayer :- } \neg \text { rich. }\} \\
& \text { (unemployed) tom } \quad=\{\text { arich. }\}
\end{aligned}
$$

The dependency graph $D G_{\text {tom }}$ is shown in Figure 3.2. Notice that the program $P_{\text {tom.taxPayer }}$ is stratified, while $P_{\text {tom.citizen }}$ is not, as the graph $D G_{\text {tom.citizen }}$ (which is equal to $D G_{\text {tom }}$ ) contains a cycle with two complementary literal symbols, namely citizen and $\neg$ citizen.

We are now ready to single out a convenient evaluation order of the rules of a stratified program, by devising a total ordering called stratification .

Given $D G_{o . p}$, let us build a new, cycle-free graph $\overline{D G}_{\text {o.p }}$ obtained by collapsing each strongly connected components of $D G_{\text {o.p }}$ into a single node of $\overline{D G}_{o . p}{ }^{2}$. There exists a topological sort $\left\langle C_{1}, \ldots, C_{n}\right\rangle$ of the nodes of $\overrightarrow{D G}_{o . p}$ (i.e., such that if there is

2 accordingly, there is an arc $\left(C_{i}, C_{j}\right)$ in $\overline{D G}_{o, p}$ if there exists at least an arc $\left(n_{i}, n_{j}\right)$ in $D G_{o . p}$ where $n_{i} \in C_{i}$ and $n_{j} \in C_{j}$. 


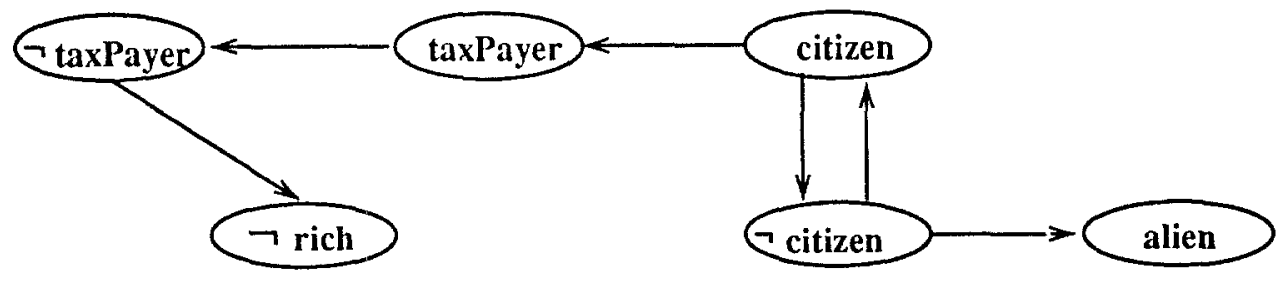

Figure 3.2: $\quad D G_{\text {tom }}$

an arc from $C_{i}$ to $C_{j}$ in $\overline{D G}_{\text {o.p }}$, then $i<j$ ), that induces a totally ordered partition $S=\left\langle S_{n}, \ldots, S_{1}>\right.$ of $P_{o, p}$, where $S_{i}$ is the set of rules whose head literal symbols are in $C_{i}$.

Given a stratified program $P_{\text {o.p }}$, the partition $S$ is called stratification and each $S_{i}$ is a stratum.

The notion of stratification is crucial for the bottom-up evaluation of a program $P_{\text {o.p }}$. Indeed, as in traditional logic programming, the process proceeds (bottom-up) from the "lower" strata to the "upper" ones. The facts that are derived for a stratum $S_{i}$ are used as input data for any stratum $S_{j}$ such that $i<j$. In addition, contradictions can be easily solved taking into account that literals derived from "lower" strata are more reliable. In fact, since in a stratified program rules with complementary head literal symbols appear in different strata, we are guaranteed that while solving a rule for $o . p$, either (i) no rule for $\neg p$ appears in $P_{o . p}$, as $o . p$ does not depend on $o . \neg p$; or (ii) all rules for $o . \neg p$ have been already solved as they are in "lower" strata. Hence, contradictions can be solved by simple set difference.

The stratified naive algorithm for OL programs is shown in Figure 3.3

Example $3.5 \quad \mathrm{~A}$ stratification for $P_{\text {som.taxPayer }}$ is $\langle\{\neg$ rich. $\},\{\neg$ taxPayer :- $\neg$ rich. $\},\{$ taxPayer. $\}\rangle$. Given such a stratification, the stratified algorithm follows the computation order intuitively shown in example 3.1.

\section{BQM Architecture}

As mentioned in the introduction, the Abstraction Layer provides a top-down evaluation of general LOCO programs. However, the actual execution is supported by a dedicated Warren Abstract Machine. Hence, to this respect, the main task of the AL is to translate a LOCO program into a persistent description of the objects, generate WAM code realizing the application, and manage the WAM execution.

The $\mathrm{BQM}$, in turn, is devoted to the bottom-up evaluation of a meaningful class of LOCO programs, that we called stratified. The general framework in which AL and $\mathrm{BQM}$ interact is shown in Figure 4.1: whenever a stratifed (sub)query is found during the top-down WAM evaluation of a query, the BQM is invoked for its bottom-up execution. The result of such a computation is then returned (one tuple at-a-time) to the WAM.

Two main distinct blocks constitute the $\mathrm{BQM}$ architecture: the Static Compiler and the Run-Time Environment, interfaced through the BQM Metaschema. Given that 
The Stratified Naive Algorithm

INPUT: a query $\operatorname{o.p}(\bar{X})$ and a stratification $\left\langle S_{1}, \ldots, S_{n}\right\rangle$ for the program $P_{\text {o.p }}$ OUTPUT: the answer to the query

METHOD: the algorithm computes ordinately the fix-point of cvery stratum $S_{i}$ by mcans of the functions fixpoint and evalRules.

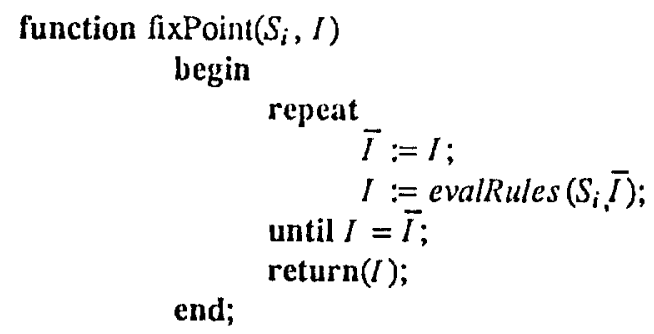

function cvalRules $\left(\left\{r_{1}, \ldots, r_{m}\right\}, I\right)$

begin

for each rulc $r_{i}$ do

let $o^{\prime}, p^{\prime}(t)$ be derived by $r_{i}$ with the facts available in $I$ if $I$ docs not contain the complementary of $o^{\prime} . p^{\prime}(t)$ then

end $I:=I \cup\left\{o^{\prime} . p^{\prime}(t)\right\}$

end;

begin \{main\}

$I:=\varnothing$

for $i:=1$ to $n$ do

$I:=\operatorname{fixPoint}\left(S_{i}, I\right)$

end.

return $(\mid o . p(l) \in I$ s.t. unifies with $o . p(\bar{X}) \mid)$;

Figure 3.3

most of the intensional information (clauses, classes, etc) is rather stable, the aim of the static analysis is to provide the run time environment with a suitably compact representation of such a knowledge. In particular - given the information gathered by the AL compiler into the $A L$ metaschema (i.e. classes' hierarchy, clauses, rules, etc.) the Static Compiler recognizes all stratified programs and stores in the BQM metaschema the result of its compilation.

The BQM Metaschema is the natural interface between the Static and the RunTime Environment: it is constructed by the former and consulted by the latter. The main goal of the Metaschema design has been to allow a straightforward retrieval of 


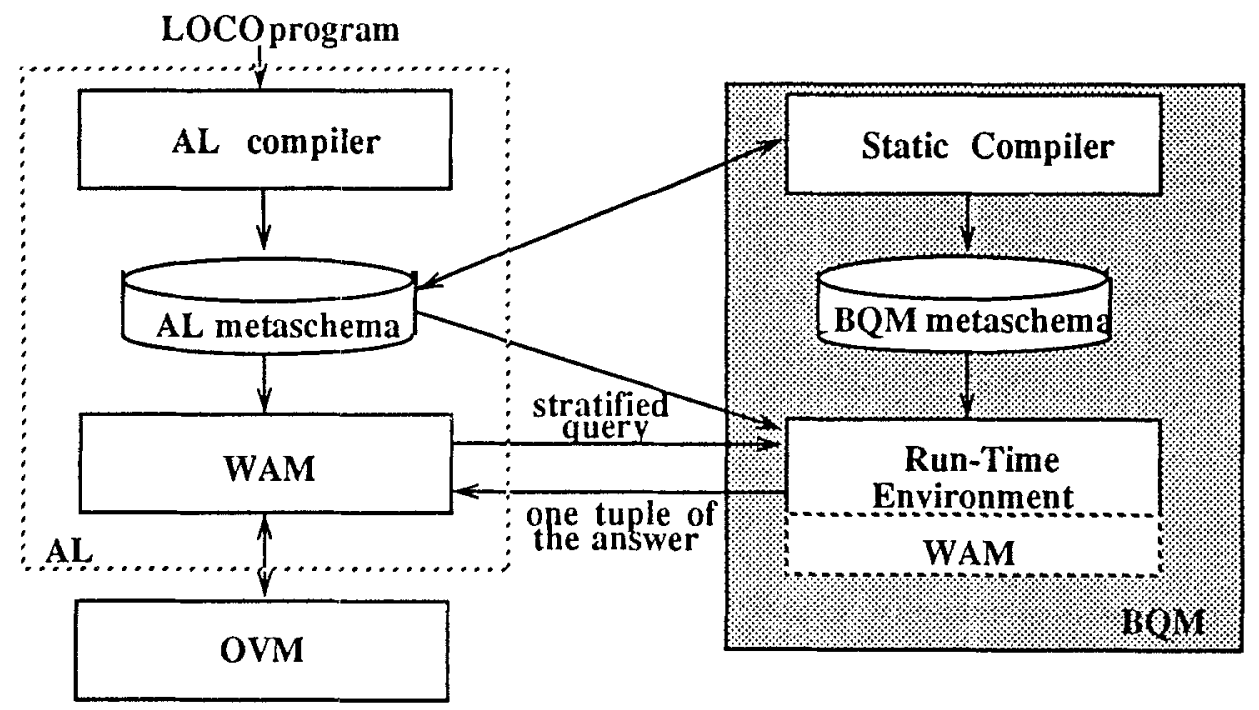

Figure 4.1: Architecture of the Basic Query Machine

programs and their stratifications.

The BQM Run-Time Environment is automatically and transparently activated by the WAM for each query on a stratified program. By using the $\mathrm{BQM}$ and $\mathrm{AL}$ Metaschema, it retrieves the LOCO program, produces an equivalent flat DATALOG program, and performs an optimized bottom-up evaluation. The crucial advantage of such a choice is that it allows the application of already consolidated optimization techniques for deductive databases. The mapping is rather straightforward and it is shown in Appendix 2.

As to the AL-BQM coupling, one of the main concerns is that the WAM, as top-down machine, consumes one tuple at a time, fetching new tuples on backtracking, while the BQM, as bottom-up machine, computes the whole answer at once. As we shall see, a simple mechanism will allow the BQM to save the computed answer and to return each tuple on WAM demand.

The remainder of this section gives more details about the BQM modules and the $\mathrm{AL}-\mathrm{BQM}$ interaction.

\subsection{The Static Compiler}

The goal of the Static Compiler is threefold: i)for each literal symbol o.p, determine if the associated program $P_{\text {o.p }}$ is stratified, ii)for each stratified program, save the stratification along with enough information for a subsequent fast retrieval of the program itself, and iii)modify the AL metaschema by building up rules that allow the WAM to invoke the $\mathrm{BQM}$ only for stratified subprograms.

As a first task, the Static Compiler builds a compact symbolic representation of the whole $\mathrm{KB}$ based on the notion of dependency graph. Clearly, the analysis is restricted to the intentional part of the KB (a more precise distinction between classes and 
instances in the framework of LOCO implementation is reported in Appendix 2).

In order to determine the sets of mutually recursive literal symbols (called clusters), the strongly connected components of the dependency graph are singled out, by using the Tarjan algorithm [Tar72]. Then, the condition of stratification of a program $P_{o . p}$ is checked, by verifying the absence of complementary literal symbols in each cluster of $P_{o . p}$. The relevant information concerning the recognized stratified programs are saved in the $\mathrm{BQM}$ metaschema. It is composed by two relations, namely bqmPred and dependsOn. The first relation describes all stratified literal symbols while the second one represents, in a compact way, the depends-on relation.

At this point the Static Compiler sets up the activation of the Run-Time Environment through the mechanism of AL external predicates. Such a mechanism represents the standard interface between the AL and external modules, allowing to associate a non-AL function to a predicate. In our case, the extemal unary predicate $b q m$ constitutes the interface between the AL and the Run-Time Environment. In particular, for each o.p such that $P_{o . p}$ is stratified, the Compiler replaces the result of the AL compilation for $p$ at the object $o$ with the simple meta rule:

$$
p(\bar{X}):-\operatorname{bqm}(p(\bar{X})) \text {. }
$$

As a consequence, the subsequent WAM code generation will produce a call to the main entry of the Run-Time Environment, whenever this is appropriate.

\subsection{The Run-Time Environment}

The BQM Run-Time Environment is activated by the WAM and is composed by two main blocks under the overall control of the BQM manager (see Figure 4.2): the Dynamic Compiler, that produces a (optimized) flat DATALOG program with negation for the query resolution; the Executor, a fix-point machine currently implemented on the WAM;

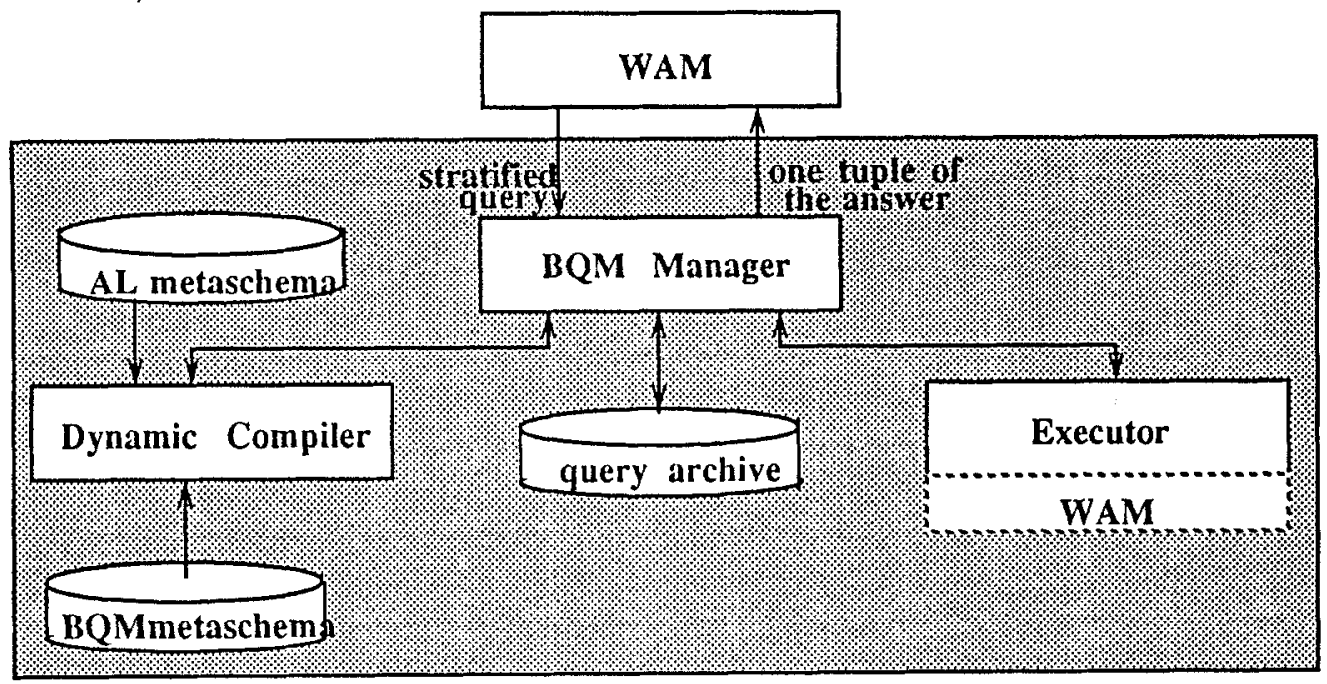

Figure 4.2: Architecture of the Run-Time Environment 
The Run-Time Environment is invoked in three possible cases:

first call

a query on the literal symbol o.p with binding pattern $b$ is fired for the first time. In this case, the BQM manager activates the Dynamic Compiler for the dynamic compilation and optimization. Then, the Executor first produces WAM code for the implementation of the fix-point of the optimized program and the construction of the whole answer relation, and then invokes the WAM for the actual execution. Thereafter, a cursor on the answer relation is created and its first tuple is returned to the calling WAM environment. Additionally, the WAM code for the triple $\langle 0, p, b\rangle$ is saved in the Query Archive.

\section{backtracking}

a query on o.p is still active, but the set of tuples of the (already computed) answer have not been all consumed. This case occurs during the top-down WAM execution of a conjunction like ...o 1.p(X),X.q(Y)..., where $o 1 . p(X)$ has been solved by the BQM. In fact, each time that $X . q(Y)$ backtracks, a new tuple of the answer to the query $o 1 . p(X)$ is required to the $\mathrm{BQM}$. By means of a valid cursor associated to the query, the $B Q M$ is able either to return a new tuple from the answer relation, or to fail if no more tuples are available.

archived query

a query on $o . p$, with a given binding pattern, has been previously (dinamically) compiled, and then archived. Thus, the $\mathrm{BQM}$ manager immediately retrieves from the Query Archive (see Figure 4.2) the address of the corresponding WAM code, and requires its execution to the WAM with the actual query binding. As for a 'first call', the first tuple is returned. A typical situation is represented by a conjunction of the form ... 1.p $(X, Y), o 2 . q(Y, Z) \ldots$, where the subquery $o 2 . q(Y, Z)$ is repeatedly passed (from the WAM) to the BQM, each time with a different binding for the variable $Y$. But, since the binding pattern is always the same, the WAM code for this query (with the given binding pattern) has been generated just the first time and then archived, so that no further dynamic compilation is required.

The next subsections contain a description of the two main blocks of the RunTime Environment.

\subsubsection{Dynamic Compiler}

Given a query $o \cdot p(\bar{X})$, where $\bar{X}$ is a list of (possibly bound) arguments, and $o$ is an object, the main goal of the Dynamic Compiler is threefold: i)load the program $P_{o . p}$; ii) flat the program $P_{o . p}$ into a stratified DATALOG program with negation as failure; iii)optimize the resulting program for an efficient bottom-up execution.

The program loading is supported by the $B Q M$ Metaschema relations for identifying the necessary rules, and by the AL Metaschema for the actual rule retrieval.

Afterwards, the rules are converted into an internal $B Q M$ format, according to the technique presented in Appendix 2.

At this point, existing techniques for efficient bottom-up implementation of stratified DATALOG programs can be applied. To this aim, some rewriting 
techniques, derived from deductive databases [SDMPDN87], based on the magic and the counting [BMSU86,BR87b,SZ87,SZ89] methods, are used. Such a rewriting produces two groups of rules: the first one exploits the binding propagation by computing sets (called magic sets) of facts that are relevant for the query evaluation; the second group contains the original rules opportunely modified so that their evaluation is restricted by such sets. Safety issues are also taken into account by a careful ordering of the goals in the rules. The same techniques have been succesfully employed in the previous ESPRIT project KIWI [KIWI88], as well as in the LDL [NT88] and NAIL! [MUV86] systems.

\subsection{Executor}

The Executor is based on a bottom-up evaluation technique which results from the combination of the semi-naive method [BR87a] with the query/subquery approach [Vie86]. The advantage of the semi-naive method w.r.t. a pure (naive) bottom-up computation is that of cutting off a number of duplicated computations that are performed by the naive strategy, where the same tuples may be recomputed over and over. On the other hand, the query/subquery strategy improves on the straight bottom-up computation by allowing binding propagation.

As it has been already pointed out, the WAM is, for the current prototype, the 'final' target (or executor) of the $\mathrm{BQM}$ programs. Hence, the main task of the Executor is to enforce, on a top-down evaluator (i.e. the WAM), a bottom-up execution. The technique is well known and it is based on a careful use of some procedural features of Prolog, eg. rule ordering, cut and fail, asserting and retracting facts and suitable flags. A detailed description of such a technique can be found in [SDMPDN87].

Clearly, besides of the generation of the appropriate WAM code, the module is also in charge of the final activation of the WAM interpreter.

\section{References}

[ABW88] Apt, K., Bair, H., and Walker, A., "Towards a Theory of Declarative Knowledge", Foundations of Deductive Databases and Logic Programming, Minker, J. (ed.), Morgan Kaufman, Los Altos, 1987, pp. 89-148.

[Ah191] Ahlsen, M., Johannesson, P., Laenens, E., Leone, N., Rullo, P., Rossi, G., Staes, F., Tarantino, L., Van Beirendonck, L., Van Cadsand, F., Van Sant, W., Van Slembroeck, J., Verdonk, B., Vermeir, D. (ed.), "The KIWIS Knowledge Base Management System", in Proceedings of the third Int. conference CAiSE'91, Trondheim, Norway, may 1991.

[AJ90] Ahlsen, M., and Johannesson, P., "Contracts in Database Federations" in Int'l Working Conf. on Cooperating Knowledge Based Systems, Keele, Springer, 1990

[BMSU86]Bancilhon, F., Maier, D., Sagiv, Y., and Ullman, J. D., "Magic Sets and Other Strange Ways to Implement Logic Programs", Proceedings of the 5th ACM SIGMOD-SIGACT Symp. on Principles of Database Systems, 1986. 
[BR87a] Balbin, I., and Ramamohanarao, K., "A Generalization of the Differential Approach to Recursive Query Evaluation", Journal of Logic Programming, 4(3), pp. 259-262, 1987.

[BR87b] Beeri, C. and Ramakrishnan, R., "On the Power of Magic", in Proc. 6th ACM SIGMOD-SIGACT Symp. on Principles of Databases Systems, 1987. pp. 269-283.

[GL90] Gelfond, M. and Lifschitz, V. "Logic Programs with Classical Negation", Proc.of 7th ICLP, Jerusalem, 1990, pp 579-597

[GS90] Greco, S. and Saccà, D. "Negative Logic Programs", Proc. of North American Logic Programming Conference, 1990.

[KIWI88] KIWI Team, A System for Managing Data and Knowledge Bases, ESPRIT Project 1117, in Proceedings of the ESPRIT Technical Week, North-Holland eds., 1988

[KIWIS89]The KIWIS team, "The KIWI(s) project: past and future", in Proc. of 6th ESPRIT Conf., 1989, pp. 594-603

[KS90] Kowalski, R.A. and Sadri,. F. "Logic Programs with Exceptions ", Proc.of 7th ICLP, Jerusalem, 1990, pp 598-616

[Lae90] Laenens, E., "Foundations of ordered logic", Ph.D. thesis, Univ. of Antwerp, 1990.

[LMRR91]Leone, N., Mecchia, A., Rossi, G. and Rullo, P., "Revised Design Documentation of the Basic Query Machine", Tech. Report BQM5/91, ESPRIT project P2424 KIWIS, 1991.

[LR91] Leone, N., and Rossi, G., "Well-founded Scmantics and Stratification for Ordered Logic Programs", submitted for publication, 1991

[LSV90] Laenens, E., Saccà, D., and Vermeir, D., "Extending Logic Programming", Proc. of ACM SIGMOD, May 1990.

[LV90] Laenens, E., and Vermeir, D., "A Fixpoint Semantics for Ordered Logic", Journal of Logic and Computation, vol.1, N.2, december, 1990, pp. 159185.

[LVVS90] Laenens, E., Verdonk, B., Vcrmeir, D., and Saccà, D., "The LOCO Language: Towards an Integration of Logic and Object Oriented Programming". in Proc. of the Workshop on Logic Programming and NonMonotonic Logic, Austin, Texas, 1990, pp. 62-72

[MUV86] Morris, K.1, Ullman, J.D., and Van Gelder, A. "Design Overview of the Nail! system" Third Int'l Conf. on Logic Programming, pp. 554-568

[NT88] Naqvi, S. and. Tsur, S. A Logical Data Language for Data and Knowledge Bases, Computer Science Press, New York, 1988.

[SDMPDN87]

Sacca' D., Dispinzeri M., Mecchia A., Pizzuti C., Del Gracco C., Naggar P. The Advanced Database Environment of the KIWI System, Database Engincering, Vol. 10, No. 4, Dec. 87 
[SLT90] Staes, F., Laenens, E., and Tarantino, L., "Towards a Flexible Interaction Environment for Knowledge Bases", in Proc. of 1990 IEEE Workshop on Visual Languages and Computing, Skokie, IL, October, 3-6, 1990.

[STVDT91]Staes, F., Tiems, B., Van Cadsand, L., D'Atri, A., and Tarantino, L., "Revised Design Documentation of the User Interface Prototype", Tech. Report UI4/91, ESPRIT project P2424 KIWIS, 1991

[SZ87] Sacca, D. and Zaniolo, C., "Magic Counting Methods", Proceedings of the 1987 ACM SIGMOD conference, 1987, pp. 49-59

[SZ89] Sacca, D. and Zaniolo, C., "The Generalized Counting Method of Recursive Logic Queries for Databases", Theoretical Computer Science, no. 62, Nov. 1989, pp. 187-220.

[Tar72] R. Tarjan, Depth First Search and Linear Graph Algorithms, SIAM J. Comput., Vol. 1, No. 2, 1972

[Tou86] Touretzky, D.S., The Mathematics of Inheritance Systems, Pitman London, 1986.

[Van89] Van Gelder, A., "The Alternating Fixpoint of Logic Programs with Negation" Proc. ACM Symp. on Principles of Database Systems, 1989.

[VGRS88] van Gelder A., Ross, K. and Schlipf, J.S., "Unfounded Sets and WellFounded Semantics for General Logic Programs", Proc. ACM SIGMODSIGACT Symp. on Principles of Database Systems, March 1988, pp 221-230.

[Vie86] Vieille, L., "Recursive Axioms in Deductive Databases: The Query/Subquery Approach", Proc. of the First Int. Conf. on Expert Database Systems, Charleston, 1986, pp. 253-268.

[VL90] Vermeir, D., and Laenens, E., "Advanced Knowledge-Base Environments for Large Database Systems", Knowledge-Based Systems, vol.3, n.4, 1990, pp. $215-220$ 


\section{Appendix 1: Stratified Programs with Extended Literals}

Because of extended literals, the program $P_{0}$ does not reduce to the rules coming from $O$ and its super-objects, but has to include a number of rules coming from completely unrelated objects. In order to avoid the collection of totally useless rules, we try to foresee which objects of the $\mathrm{KB}$ can be involved in the computation.

To this aim, we first define the range of an extended literal symbol $X . q$ as the set of objects equals to: i) $\{o\}$, if $X$ is equal to the object $o$; ii) the set of all objects that own or inherit a rule for $q$, otherwise.

Let us consider, as an example, the following EOL program.

$$
\begin{aligned}
& o^{\prime}=\{p(X):-q(Y), Y . r(X) \text {. } \\
& \neg w(X):-s(X) \text {. } \\
& w(X):-p(X) .\} \\
& \left(o^{\prime}\right) 0=\{\neg p(X):-s(X) \text {. }\} \\
& \text { (o) } o_{1}=\left\{q\left(\bar{o}_{1}\right) . s(a) . s(b) .\right\} \\
& \bar{o}=\{r(X):-p(X) .\} \\
& \text { (o) } \bar{o}_{1}=\{p(a) .\} \\
& \hat{o}=\{r(X):-p(X) .\}
\end{aligned}
$$

According to the above definition, the range of the extended literal $Y . r(X)$, appearing in the rule for $p$ at $o^{\prime}$, is equal to $\{\bar{o}, \hat{o}\}$ as they are the only objects with rules for $r$.

At this point, the program $P_{o}$ associated to the object $o$ can be identified as the set of rules that belong to: i) $O$ and all its super-objects; ii)the programs $P_{o^{\prime}}$ for each $o^{\prime} \neq 0$ in the range of each extended literal symbol X.q appearing in $P_{0}$.

To remark the difference between literal symbols defined on different objects, a generic literal symbol $p$ appearing in a rule of $P_{o}$ will be denoted by a dot-notation like $t . p$ where $t=o$ if the rule is determined by condition i) above, or $t=0^{\prime}$ when given by condition ii).

Let us consider again the objects defined in the previous example. In order to determine the program $P_{o}$, we first take all rules at $o$ and $o^{\prime}$. Thereafter, the same process is iterated for all objects in the range of $Y . r(X)$, yielding, after the inclusion of $P_{\bar{o}}$ and $P_{\delta}$, the following program:

$$
\begin{array}{lll}
o . w(X):-o . p(X) . & o . \neg w(X):-o . s(X) . \\
o . p(X):-o . q(Y), \bar{o} . r(X) . & o . p(X):-o . q(Y), \hat{o . r}(X) . & 0 . \neg p(X):-o . s(X) . \\
\overline{o . r}(X):-\bar{o} . p(X) . & \hat{o . r}(X):-\hat{o} . p(X) .
\end{array}
$$

Notice that the instantiation of the extended literal $Y . r(X)$ has generated two rules for $o . p$, one for each object in its range.

Now, it is not difficult to see that the dot-notation introduced above for denoting a generic literal symbol extends the validity of all definitions given for the stratification, to the presence of extended literals. Thus, the depends-on relation for the program $P_{o}$ is represented by $(o . w, o . p),(o . \neg w, o . s),(o . w, o . \neg w),(o . \neg w, o . w)$, $(o . p, 0 . q),(o . p, \bar{o} . r),(o . p, \hat{o} . r),(o . p, o . \neg p),(o . \neg p, o . s),(\bar{o} . r, \bar{o} . p),(\hat{o} . r, \hat{o} . p)$.

Consequently, the dependency graph looks like in Figure Notice that the dependency graph for $o$ includes the dependency graphs for $\bar{o}$ and $\hat{o}$. 


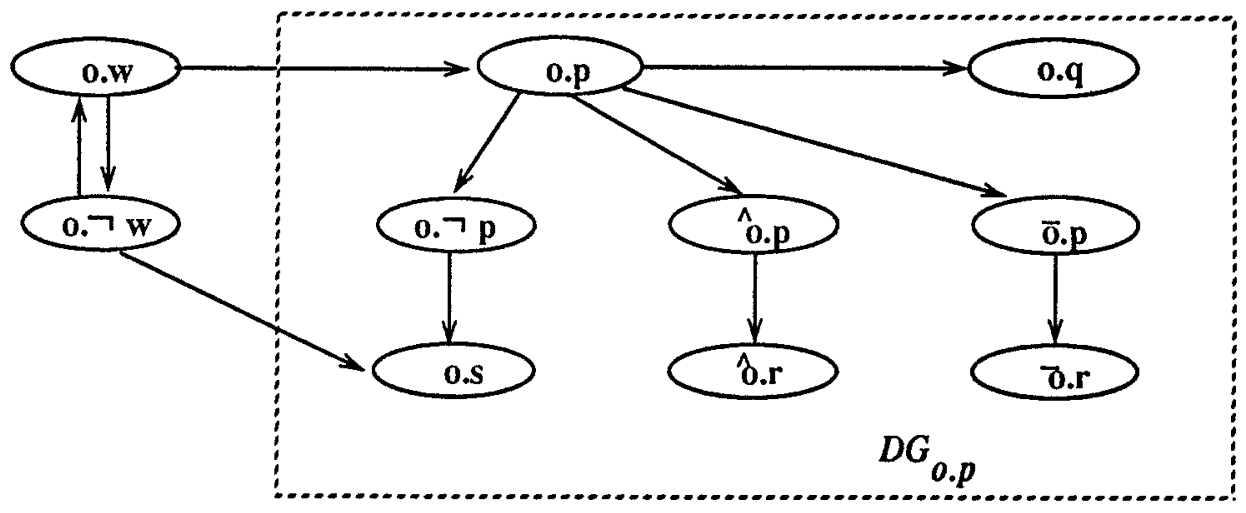

Now, just as shown in Section 3, the programs needed to solve any query on $o$ can be still singled out from the graph, and the stratification can be checked against the dependency graph by taking into account that two complementary literal symbols are of the form o.p and $\neg$ o.p. In the example, the programs $P_{o . w}$ and $P_{o . \neg w}$ are not stratified. In fact, the dependency graphs $D G_{o . w}$ and $D G_{o . \neg w}$, which coincide with $D G_{o}$, contain a cycle with $o . w$ and $o . \neg w$. On the other hand, the program $P_{o, p}$ is stratified, as it is clear from the dependency graph $D G_{o . p}$ shown in figure.

\section{Appendix 2: From EOL to DATALOG}

It has already been shown in [LMRR91,LR91] that a stratified OL program can be constructively transformed into an equivalent (stratified) DATALOG program. In this Appendix, we present, in a step-by-step fashion, the transformation as it has been devised for an actual implementation of EOL programs.

As a preliminary consideration, we note that, for efficiency reasons, it is convenient to introduce a distinction between the class and instance objects. An instance is an object that i)has only facts in its definition, ii)has no sub-object, and iii)has only one super-object, which is its class. All other objects are classes. Hence, programs associated to instances are only slightly different from those of their classes. Notice that such a distinction follows the dualism between schemes and instances of the traditional database approach, with the main advantage of limiting the analysis of the $\mathrm{KB}$ only to classes.

Now, let us assume that we have identified the (stratified) program $P_{o . p}$ needed to solve a query onto $0 . p$, that the program has been divided into cluster of mutually recursive predicates, and that the associated stratification is known. Further, for semplicity, we suppose that each cluster is associated to a unique identifier $c$.

To begin with, all rules of $P_{o . p}$ are adorned such that each literal $\hat{o} . q(\bar{X})$ appearing in $P_{o . p}$ is rewritten as $q^{c}(\hat{o}, \bar{X})$, where $c$ is the identifier of the cluster $\hat{o} . q$ belongs 
to and the object $\hat{o}$ has become the first argument of the literal. However, in the following, we will omit the cluster identifier whenever no ambiguity arises. Further, notice that $p$ and $\neg p$ are considered different positive literal symbols.

The first step is to generalize the rules so that the same program holds for a class and all its instances. Thus, a rule

is rewritten as

$$
q(\hat{o}, \bar{X}):-\operatorname{body}(\hat{o}, \bar{X}) .
$$

$$
q(S, \bar{X}):-\operatorname{body}(S, \bar{X}) \text {, instance }(S, \hat{o}) \text {. }
$$

where instance $(S, \hat{o})$ is a built-in predicate associating to the class $\hat{o}$ the set $S$ of its instances.

The second transformation aims to code into the clauses the overruling mechanism. Each rule of the form

$$
q(S, \bar{X}):-\operatorname{bod} y(S, \bar{X}), \text { instance }(S, \hat{o}) \text {. }
$$

such that $P_{o, p}$ contains a rule $\neg q(S, \bar{Y}):-\ldots$, instance $(S, \bar{o})$. where $\hat{o}$ is a super-object of $\bar{o}$, and $\neg q$ denotes the complementary of $q$, is rewritten as

where not is interpreted as negation as failure.

$$
q(S, \bar{X}):-\operatorname{body}(S, \bar{X}), \text { instance }(S, \hat{o}), \operatorname{not}(\neg q(S, \bar{X})) \text {. }
$$

The above rewritten rule simply states that a fact $q(t)$ can be derived only if the complementary fact $\neg q(t)$ cannot be derived. Notice that such a transformation fully captures the idea of overruling for stratified EOL programs.

An analogous approach is used for dealing with simple cases of non-stratified programs, where the non-stratification is induced by facts defined on the instances. The idea is that, whenever a rule $q(S, X)$ :-body $(S, X)$,instance $(S, o 1)$ is contradicted by some facts for $\neg q$ declared at any instance of $o 1$, then it is rewritten as

$$
q(S, X):-\operatorname{body}(S, X) \text {, instance }(S, 01) \text {, not }\left(\neg q_{\text {base }}(S, X)\right) \text {. }
$$

where $\neg q_{\text {base }}$ will match only the facts for $\neg q$ defined on the instances.

A further transformation involves the management of extended literals. Given a rule $q^{c}(S, X):-\cdots, Y . t(Z), \cdots$. let $o_{1}, \ldots, o_{i}$ be the objects in $\operatorname{range}(Y . t(Z))$ and let $c_{1}, \ldots, c_{i}$ denote the clusters of respectively $o_{1} . t, \ldots, o_{i} . t$. Then the rule is rewritten as:

$$
\begin{aligned}
& \frac{q}{c}^{c}(S, X):-\cdots, t^{k}(Y, Z), \cdots \\
& t^{k}(S, Z):-t^{c}(S, Z) . \\
& \cdots \\
& \bar{t}^{k}(S, Z):-t^{c_{i}}(S, Z) .
\end{aligned}
$$

where $\bar{t}$ is a new literal symbol, added for avoiding a duplication of the original rule (i.e. a rewriting for each $t^{c_{i}}$ ). Moreover, if any $t^{c_{i}}$ is mutually recursive with $q^{c}$ (i.e. any $c_{i}=c$ ), then $\bar{t}$ gets the cluster identifier $c$ (i.e. $c=k$ ) and becomes mutually recursive with $q^{c}$, otherwise $\bar{t}$ gets a new cluster identifier.

The transformation of the stratified EOL program $P_{\text {o.p }}$ into an equivalent DATALOG program is now complete. It is also possible to see that the resulting program is stratified (in classical sense [ABW88]) and that its stratification can be derived from that of the original EOL program. 
As a matter of fact, from one hand, the insertion of literals defined only by facts (e.g. instance) cannot affect the stratification $S$ of the EOL program; on the other hand, the usage of not $(q)$ in the rules for $\neg q$ implies that such rules belong to a stratum higher than the stratum containing the rules for $q$ : because of overruling, this fact is already granted in $S$. Finally, the addition of rules for the new literal symbol $\bar{t}^{k}$ modifies $S$ in a very simple way: the rules for $\bar{t}^{-k}$ are either in the same stratum $S_{q}$ of the rules for $q^{c}$ if $k=c$, or in a new stratum that must immediately precede $S_{q}$. 